\title{
RANGE-BASED DATA GATHERING ALGORITHM WITH A MOBILE SINK IN WIRELESS SENSOR NETWORKS
}

\author{
A. Alhasanat ${ }^{1}$, K. Alhasanat ${ }^{2}$ and M. Ahmed ${ }^{3}$ \\ ${ }^{1}$ Department of Computer Engineering, Al-Hussien Bin Talal University, Jordan. \\ ${ }^{2}$ Department of Computer Science, Middle East University, Jordan. \\ khaled \\ ${ }^{3}$ Department of Computer Science, Aqaba University of Technology, Jordan.
}

\begin{abstract}
Wireless Sensor Networks (WSNs) have been emerged in many important aspects in the real world, such as industry, agriculture, and military applications. As the main challenge that WSNs facing is the energy consumption, it is necessary to investigate the suitability of using mobile sinks for data collection in these networks. In this paper, therefore, a new data gathering technique with mobile elements referred to as Intersection Point of Communication Ranges (IPCR) is proposed. The IPCR algorithm endeavours to compute the optimal trajectory of the mobile sink for which the data collection latency is reduced. Simulation results presented in this study showed that the IPCR algorithm has achieved the optimal Travel Sales-Man Problem algorithm. In addition, the IPCR algorithm outperformed the Connectivity Based Data Collection $(C B D C)$ algorithm in terms of data gathering latency and network throughput.
\end{abstract}

\section{KEYWORD}

Wireless Sensor Networks, Data Collection Algorithm, Received Signal Strength (RSS) and Mobile Sink.

\section{INTRODUCTION}

Recently, the advancement of Microcontrollers and wireless communication technologies have led to the development of low cost, low-power and multifunctional sensor nodes known as Wireless Sensor Networks (WSN). These sensor networks consist of a large number of sensor nodes which are responsible for data sensing, processing, and communication [1]. Currently, WSNs are substantially considered for a wide spectrum of applications, for instance battlefield surveillance, habitat and traffic monitoring, security systems, etc.

Unfortunately, the tiny size of the wireless sensor nodes limits the processing, storage, and communication capabilities of these networks. More importantly, these networks are also suffering from restricted energy resources, as they often battery-powered and difficult to recharge, particularly within remote environments of inaccessible locations. Since these nodes consume most of their energy for data communication, energy conservation represents a challenging design issue for prolonging the lifetime of these networks [2]. 
In literature, dozens of techniques have been studied in an attempt to reduce power consumption due to data gathering in WSNs. Accordingly, data gathering can be categorized into traditional routing algorithms and mobile collector techniques, such as [3], [4], [5], [6], [11]. With the former approach, single-hop or multi-hop communication is needed to collect data from the source node to the sink node. With single-hop communication, distant nodes require higher transmission power than others and then will run out of their energy rapidly. Although multi-hop communication can be used to solve this problem, additional burdens will be incurred on intermediate nodes, particularly nodes located near the sink node. Methods such as Low Energy Adaptive Clustering Hierarchy (LEACH) [15] and Power-Efficient Gathering in Sensor Information Systems (PEGASIS) [16] were developed in an attempt to achieve consistent power dissipation. Despite that with these methods the performance of WSN is greatly improved, they still encounter the problem of limited network lifetimes.

At the other extreme, data gathering can be accomplished using mobile sink nodes, where a mobile sink moves around the sensing field and collect data from nearby nodes. In comparison with traditional routing algorithms, for mobile collectors there is no routing algorithms required and so no nodes overhead imposed. In addition, mobile collectors have reported a significant increase in the network lifetime since nodes send their data only when the mobile sink is close enough to these nodes [8], [12].

In fact, two main problems experience data gathering. The first one is the limited power supply of the mobile sink. When the path of the mobile sink is too long, then it is possible that not all data can be collected from all sensors. The second problem is represented by the data collection latency associated with low speed mobile elements. Actually, data gathering with single mobile sink leads to delay the data collection phase which sometimes exceeds the time deadline prescribed by the application under consideration. However, using multiple mobile elements has resolved this problem in spite of the additional network cost required. Moreover, adopting data gathering within environment of inaccessible areas is a challenging issue. However, it is worth mentioning that this research covers the applications where sensor locations are accessible, for example by using an autonomous mobile sinks.

This paper endeavours to design a trajectory for a mobile sink such that,

- All sensor nodes are covered given node's locations and communication range.

- The data gathering latency is minimized.

The first condition ensures that the mobile sink will traverse all nodes in order to avoid the use of multi-hop communication. This is important to increase network life time of sensor nodes since using single-hop communication for short distances will indeed reduce the energy consumption of sensor nodes. In the second condition, the data gathering latency is reduced at a given speed of the mobile sink. In fact, by satisfying this constraint, sensor nodes would have the opportunity to increase it is sensing rate from one hand, and therefore, to increase network throughput from the other hand. Reducing data gathering latency can be achieved via either increasing speed of a mobile sink or minimizing the tour length. This study focused on reducing data gathering latency via minimizing the tour length of the mobile sink.

In this paper, a new data gathering algorithm referred to as Intersection Points of Communication Range between a mobile sink and sensor nodes (IPCR for short) is proposed. The efficiency of the IPCR algorithm is assessed through simulation. A comparison between the IPCR algorithm and the optimal Travel Sale Man (TSP) algorithm is made. Results obtained by the IPCR algorithm have matched the optimal TSP algorithm. Moreover, the data gathering latency and network throughput of the IPCR are evaluated and compared with the Connectivity Based Data Collection (CBDC) algorithm [4]. The results emphasized the superiority of using the IPCR algorithm over the CBDC algorithm. 
This paper is organized as follows. In the next section, the related work is discussed. Section 3 formulates the problem statement. The IPCR algorithm is given in Section 4. The simulation results are presented in Section 5. The conclusion and future are finally drawn in Section 6.

\section{LITERATURE REVIEW}

In this section, we present some recent research works that focus on the mobility-based data gathering in wireless sensors networks (WSNs). Firstly, the mobility of sink node can be categorized as controlled, predefined and Random Mobility [4]. In the controlled mobility, mobile elements visit sensor nodes in a controlled manner which can then be changed from time to time. For example, M. Ma suggested a data collection algorithm using mobile sink called Mcollector [8]. This method makes a tour starting from the sink, traversing all sensor nodes to collect the data and then come back to the sink to upload the collected data. With this method, however, a change in the network topology will lead to a change in the path of the M-collector.

For a predefined mobility, on the other hand, the path for the mobile sink can be previously determined and cannot be changed during the network lifetime. A predefined mobility type is suitable for structural health monitoring (SHM) and infrastructure surveillance applications. An example on this type of mobility is the warning system based on WSNs for monitoring railway infrastructure [17].

When the mobile sink moves in the network without any predefined path also without any controlling capability is called random mobility. This mobility is appropriate to investigate the wild-animal behaviour. For example, it has been adapted in [18] to track zebras.

Data in WSN is further gathered using mobile base station, mobile data collector and Rendezvous techniques [14]. Mobile base station (MBS) aims to balance the energy in the network by changing the position of a mobile sink during the operation time. Then send data gathered from sensors to MBS without long term buffering. In [19], Gandham partitioned the time into rounds. MBS, when completes a collection round, changes its location in the network in order to balance the energy consumption of sensor nodes. In contrast, with the Mobile Data Collector (MDC)based solution a mobile sink moves in the network and collects the buffered data from sensor nodes using single-hop communication. In [10] R. Shah used mobile data collection called data mules. In this technique, mules move through the network to gather the data buffered in the sensors located in its communication range and transmit it to a wired network. With the two mentioned approaches, costly multi-hop wireless transmissions are avoided. However, they suffer from the increased latency, since the speed of most ME is very low. This for large sensing field yields the ME to mismatch the delay requirements of many applications. Rendezvous based approach, on the other hand, is a combination between the two mentioned approaches. The sensor nodes transmit the data to rendezvous points (RPs) using multi-hops communication. Data remain buffered until uploaded to the mobile device. It is confirmed that with the Rendezvous method, huge size of data can be collected without traversing a long distance, and hence, can achieve high bandwidth data with low communication delay [7]. A new algorithmic approach based on rendezvous points developed by G. Xing [7]. In this method, some nodes work as rendezvous points (i.e. collection points) to collect and buffer data from sensor nodes and upload it to the mobile sink when it arrived. Another example on Rendezvous point algorithms where MEs paths are constrained on the data routing tree are RP-CP and RP-UG. RP-CP aimed to find the optimal rendezvous points when the mobile sink moves along the data routing tree. While RP-CP attempted to obtain the optimal rendezvous points that achieve a desirable compromise between energy saving and travel distance for the mobile sink. Simulation results showed that these techniques reduced the energy consumption significantly and then extended the lifetime of the network Other research have focused on planning the path of the mobile sink to become a 
mechanism that can improve energy consumption and prolong the network lifetime. For instance, a new heuristic based scheme to find the optimal path for the mobile sink in order to collects the data from sensor nodes and delivers it to the sink node, was introduced in [20]. This technique ensures that the travelling time of the mobile sink is less than or equal the transit time constraints, where the transit time constraint is the time interval from data generation to data delivery to the BS. Furthermore, Maximum amount shortest path (MASP) is a new algorithm proposed by S. Gao et al [9] to maximize the data collection amount and reduced the energy consumption using optimized mapping between sub-sinks and sensor nodes. In this method, a number of mobile sinks is moving on the sensor field. Hence the sensor field is divided into regions of direct communication area (DCA) and multi-hop communication area (MCA). Sensor nodes within the DC are called sub-sinks and MCA for far sensors are called members. Each mobile sink collects data from sensor nodes while moving close to them. Because of the fixed path and speed to mobile sink, the duration time between each sub-sink and the mobile sink is fixed, so that throughput of the WSN depends on the relationship between the data collected and the number of members belonging to each sub-sink. The difficulty herein is the requirement of an efficient assignment of members to the sub-sinks in order to reduce energy consumption. MASP can support sensor networks with low density and multiple mobile sinks. As further improvement to MASP, zone partitioning-based and a distributed algorithms (MASP-D) were made in order reduce the complexity.

Utilizing communication range of sensor nodes and mobile sink to design a data gathering algorithm has not been fully considered in literature. The reason is due to the fact that there are some factors affect the communications range in WSNs such as channel fading and radio irregularity, and then modelling an accurate communication range is a trivial task. Nevertheless, cooperative MIMO (Multiple Input Multiple Output) communication, for instance, was proposed for long range transmission [21]. In this approach, a mobility aided cooperative MIMO based communication model namely MACO MIMO was developed, in which mobile sensors move to identify location and collected data from sensors and then send it to cooperative sensor or sink nodes. There are two types of sensors, the first called Listeners, and the second called Supervisors, where Listeners sense and send data to Supervisors, while Supervisors (Rechargeable supervisors reduce the workload on listeners) have more higher capacity and can move over the field. Pairing of supervisors represents a cluster of the listener sensors to share the workload in a cluster and also to easily control the cooperative distance between two cooperative supervisors. The benefit from using this model of communication is the uniform energy consumption of sensor nodes and hence sufficient average lifetime for the network [21]. However, it suffers from the additional hardware required to implement MIMO, in particular for wireless sensor nodes.

The method presented in this paper is a range based data gathering with Single Input Single Output (SISO). In addition, the Received Signal Strength (RSS) is adopted as it does not require any additional hardware and almost exist in all WSNs.

\section{Problem Formulation}

In this paper, data from $\mathrm{N}$ stationary sensor nodes need to be collected by a single node known as mobile sink. All sensor nodes are using static transmission power, i.e. same level of transmission power. The mobile sink must follow a limited path through a set of collection points in order to satisfy its energy and time constraints. In addition, in this path it is preferable to avoid any form of multi-hop communication between sensor nodes and the mobile sink, as mentioned in the introduction section. 
Let

$$
S=\left\{C_{1}, C_{2}, \ldots, C_{M}\right\},
$$

represents the collection points of the mobile sink, where

$$
C_{i}=\left\{n_{1}, n_{2}, \ldots, n_{p(i)}\right\}
$$

is the set of members of sensor nodes that belong to the collection point $C_{i}$ and $n_{p}(i)$ is the number of members in this set. Note that for a network without connectivity,

$$
|S|=M=N \text {, }
$$

in this case the mobile sink must visit the location of each sensor node to gather its data, which is the worst and unrealistic scenario. However, for a fully connected network, we have

$$
|S|=1 \text {, }
$$

This indicates that the mobile sink is required to visit one location to collect data from all sensor nodes which is the desired but uncommon scenario. In this paper we are interesting with the most realistic scenario, where

$$
1<|S|<N \text { and } N>1 \text {, }
$$

The mobile sink, in this case, has to traverse all collection points and collect data from their member nodes via single-hop communication.

Once the data collection round begins, the mobile sink begins to move from the sink node location passing through the selected collection point with a speed of q $\mathrm{m} / \mathrm{s}$. Hence, $\frac{l}{q}$ seconds is required to complete a single data collection round of length $l$. During each round, each sensor node should send its data to the mobile sink. Two approaches are suggested to organize data collection and to prevent out-of-synchronization problem.

In the first one, the sensing rate is set to $\frac{l}{q}$ seconds. The problem of out of synchronization still occurs when for instance the mobile sink is slowed down or even delayed. In the

(a)

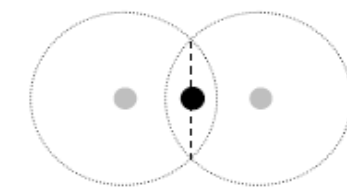

Sensor nodes

Collection points

Communication range (b)

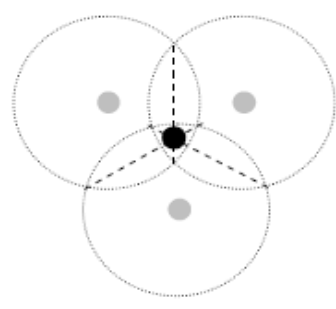

(c)

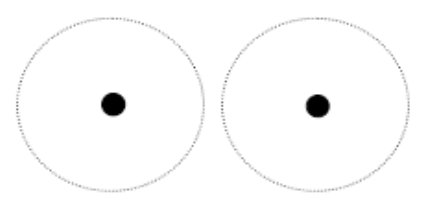

Figure 1 .Collection points determination scenarios. a) Two overlapped nodes, (b) more than two overlapped nodes and (c) isolated nodes. 
second method, Stop-to-Collect Data (SCD) algorithm [22] can be used. Hence, once the mobile sink arrived at a CP, it starts a TDMA slot for data collection. By assuming that each sensor node needs $\tau$ to send its data to the sink node on collection point, such that

$$
\tau=\frac{K}{R}
$$

for a $K$ packet size, $R$ data rate and ignoring propagation delay, the mobile sink needs to wait a TDMA slot at a collection point $\mathrm{i}$ as

$$
\operatorname{TDMA}(i)=\tau \times n_{p}(i)
$$

Therefore the total Latency of a single data collection round is computed as

$$
\text { Latency }=\frac{l}{q}+M * \max _{1 \ldots \mathrm{M}}\{T D M A(i)\}
$$

where $l$ is the path length of the mobile sink. Here, the system throughput can be calculated as

$$
\text { Throughput }=\frac{K N}{\text { Latency }}
$$

\section{The Proposed Algorithm}

Each sensor node firstly broadcasts a beacon message to determine its neighbours based on the received signal strength (RSS). Then, RSS measurements are used to compute the inter-sensor distances and so how close these nodes to each other. Consequently, each sensor can maintain a list of its neighbour IDs, which helps to provide some information about the selection of collection points of the mobile sink.

Assume that $d_{i j}$ represent Euclidean distance between nodes i and $\mathrm{j}$, and $r_{i, j}$ is the corresponding communication range between these nodes. The two nodes are connected with each other if

$$
d_{i j}<r_{i, j}
$$

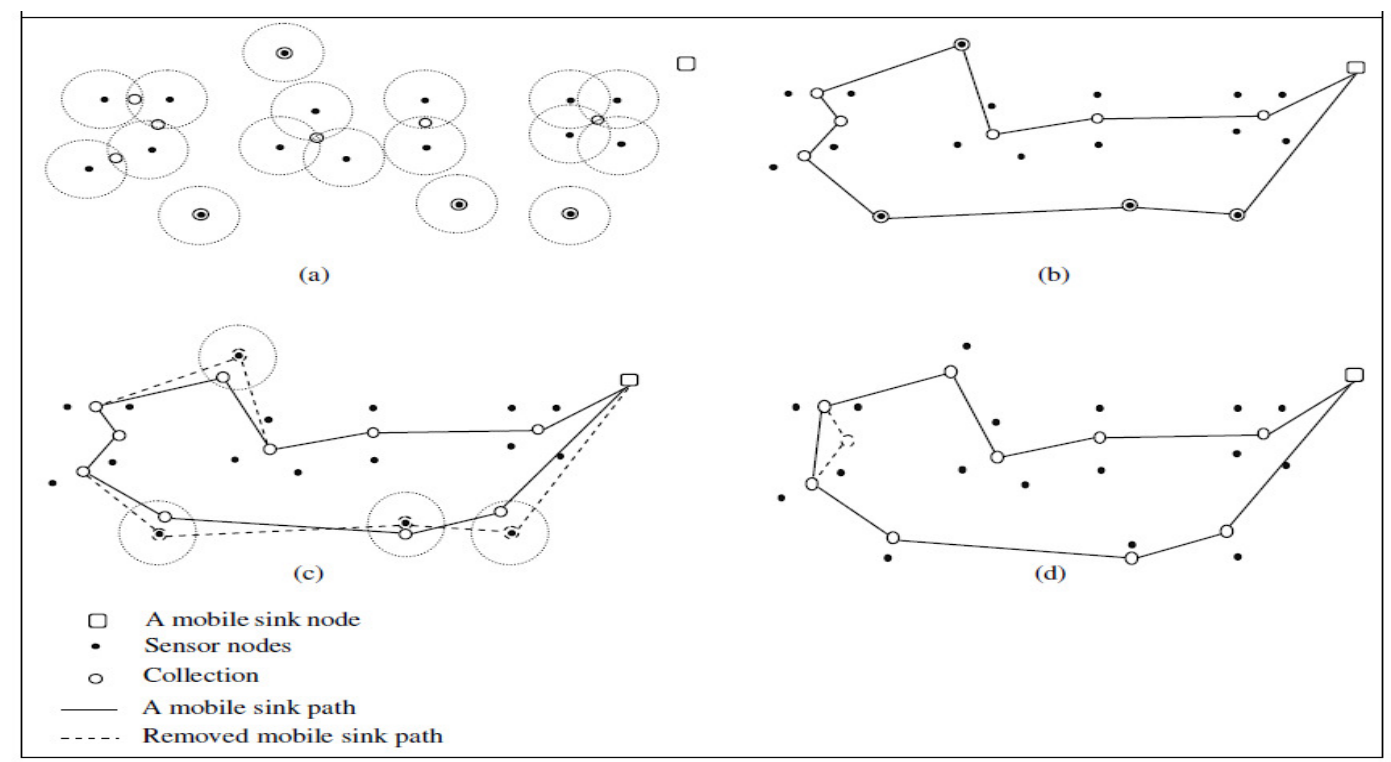

Figure. 2. Examples on a) Sensor node's communication ranges and their intersection points, b) TSP path through the intersection points (i.e. collection points), c) the path after the first refinement and d) the path after the second refinement. 
assuming that $r_{i, j}=r_{j, i}$. However, the mobile sink is required to visit an overlapped area between two or more nodes with unnecessarily that these nodes are connected to each other. Hence, communication ranges of two nodes are overlapped if

$$
d_{i j}<2 r_{i, j}
$$

Generally speaking, for m nodes with an overlapped area, there is a location for which the mobile sink has to stop and collect data. This location is determined as the centroid of this area as shown in Figure 1 For example for two nodes (Figure 1(a)), the collection is defined as the centroid of a line connecting the interconnection points of the communication ranges of the two nodes. while for more than two nodes, the collection point is determined as the intersection points of all lines connecting the intersection points of communication ranges of all nodes, as shown in Figure 1(b). While for isolated nodes, the locations of these nodes are used as collection points for the mobile sink. This is the case illustrated in Figure 1(c). After determining the collection points, Travel Sales-Man Problem (TSP) is then used to obtain the shortest path through these collection points. Once the mobile sink completes a data collection round, it uploads the data into the sink node and starts a new round.

\subsection{Path Refinement}

One refinement on the path length of the mobile sink is possible particularly on the isolated nodes for each of which there is a collection point exactly located on the same location of these nodes. However, it is fair enough for the mobile sink to visit points on communication ranges of such isolated nodes whilst minimizing the total tour length of the mobile sink. In the IPCR algorithm, a new collection of an isolated node is calculated as the centroid of a line connecting the intersection points between this node's communication range and the path line of the mobile sink. This scenario is clearly shown in Figure 2(c) for many isolated nodes.

Furthermore, it is possible that a collection point which linked to a set of sensor nodes, while these nodes were already linked by another adjacent collection points. This in turn leads to increase the path length of the mobile sink, and hence such collection points should be removed. To accomplish this task, assume that the tour of the mobile sink after computed by TSP algorithm is given as

$$
\mathrm{T}=\left\{\mathrm{C}_{1}, \mathrm{C}_{2}, \ldots, \mathrm{C}_{\mathrm{M}}, \mathrm{C}_{1}\right\}
$$

then the collection point will be removed from $\mathrm{T}$ when the sensor nodes associated with the collection point $\mathrm{Ci}$ are already associated with $\mathrm{C}_{\mathrm{i}-1}$ or $\mathrm{C}_{\mathrm{i}+1}$, such that

$$
C_{i}=\left(C_{i} \cap C_{i+1}\right) \cup\left(C_{i} \cap C_{i-1}\right)
$$

Figure 2(d) shows an example on this scenario.

\section{Perfomance Evaluation}

In this section, we study the performance of the Intersection Point of Communication Range (IPCR) algorithm. In this experiment, the trajectory of the proposed algorithm was compared with the optimal TSP algorithm obtained by the Mixed-Integer Linear Programming (MILP). In addition, the efficiency of our algorithm was compared with the Connectivity Based Data Collection algorithm (CBDC)[4] in terms of data collection latency, number of collection points and the network throughput. Networks of different levels of connectivity and varying number of sensor nodes were considered in this simulation. 


\subsection{Simulation Scenario}

\begin{tabular}{|c|c|c|}
\hline connectivity $100 \%$ & $d_{\text {th }}$ meter & $\mathbf{R S S}_{\mathrm{th}} \mathbf{d B}$ \\
\hline$\approx 0$ & 4 & -55 \\
\hline 0.001 & 7 & -60 \\
\hline 0.002 & 12 & -65 \\
\hline 0.007 & 20 & -70 \\
\hline 0.01 & 33 & -75 \\
\hline 0.1 & 54 & -80 \\
\hline 0.15 & 90 & -85 \\
\hline 0.30 & 148 & -90 \\
\hline 0.60 & 245 & -95 \\
\hline 0.90 & 404 & -100 \\
\hline
\end{tabular}

Table 1: The RSS values RSSth, the coverage area radius dth and the Network connectivity used in the this experiment. The number of sensor nodes is 300 .

A sensor field of $400 \times 400 \mathrm{~m} 2$ was considered in which the sensor nodes are uniformly and randomly distributed. The transmission rate of all sensor nodes was set to $250 \mathrm{Kbps}$. The speed of the mobile sink was set to $1 \mathrm{~m} / \mathrm{s}$. In this simulation scenario, the connectivity of sensor nodes was measured as [13]

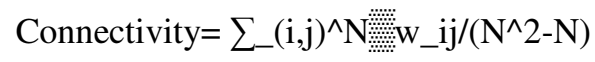

Notice that wi,j is influenced by the pair-wise RSS measurements between sensor nodes. As network connectivity severely depends on the RSS values, a range of RSS were considered and the equivalent sensor connectivity values were recorded, as listed in Table 1.

\subsection{Path Length of the Mobile Element}

In order to examine the efficiency of the new proposed algorithm (IPCR algorithm), the total path length of the mobile sink is compared with the one achieved using the optimal Travel-Sale Man (TSP) algorithm. The later was implemented using the Mixed Integer Linear Programming (MILP) [28]. As the MILP TSP is a brute force search method, it is difficult to run this algorithm for large network sizes and hence, a small network sizes were used for this comparison.

Intuitively, as the number of sensor nodes increased the path length of the mobile sink is increased, and hence IPCR with limited number of collection points will obtain a shorter path length than the TSP MILP when considering all nodes. Therefore, to provide a consistent and reasonable comparison, in the first scenario the optimal TSP algorithm was solved for all nodes and then was applied for the collection points obtained by the IPCR algorithm in the second scenario. 
Figure 3 (a) shows the path length of the mobile sink as a function of in-creasing number of sensor nodes. The most important trend from this figure is that the IPCR exhibited an identical performance in comparison with the optimal solution when the TSP MILP applied for collection points only. In addition, the two algorithms demonstrate a slight increase of the path length in comparison with TSP MILP on all nodes. This result, which is confirmed in the subsequent results, emphasis the advantage that our algorithm is highly scalable in that the path length of the mobile sink is less dependent on the number of sensor nodes. Similarly, the IPCR performs almost identical performance to the optimal TSP at a wide spectrum of communication ranges. Indeed the TSP MILP on the original nodes demonstrate a constant path length of the mobile sink as this algorithm computes the tour length on all nodes and without considering communication ranges of sensor nodes into account.

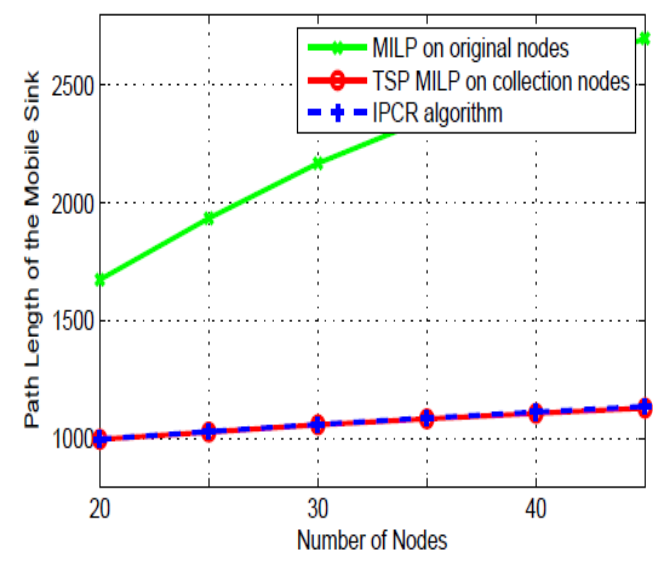

(a)

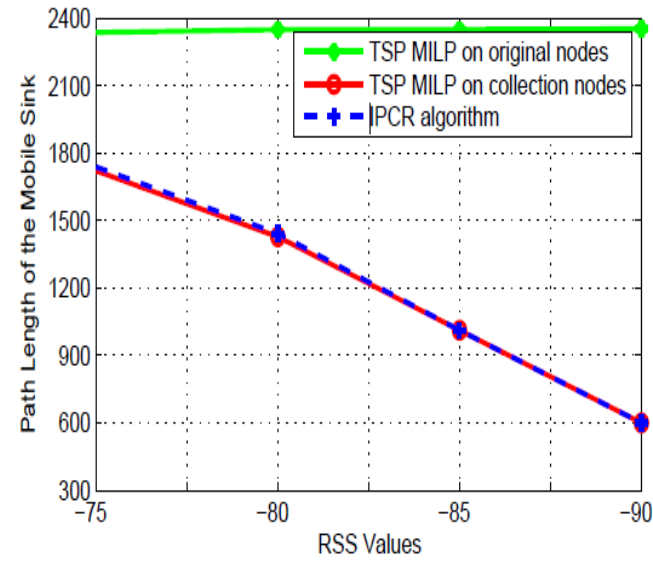

(b)

Fig. 3. Mobile sink path lengths comparison between the IPCR algorithm and the optimal Travel-Sale Man (TSP) using MILP for (a) Number of sensor nodes and (b) RSS values.

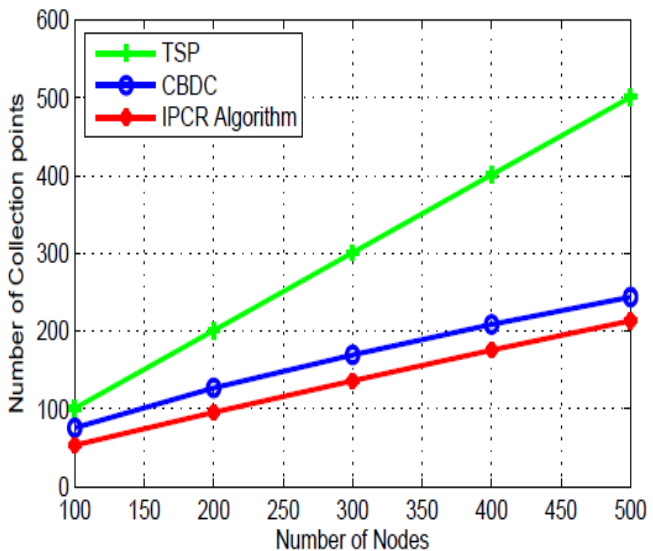

(a)

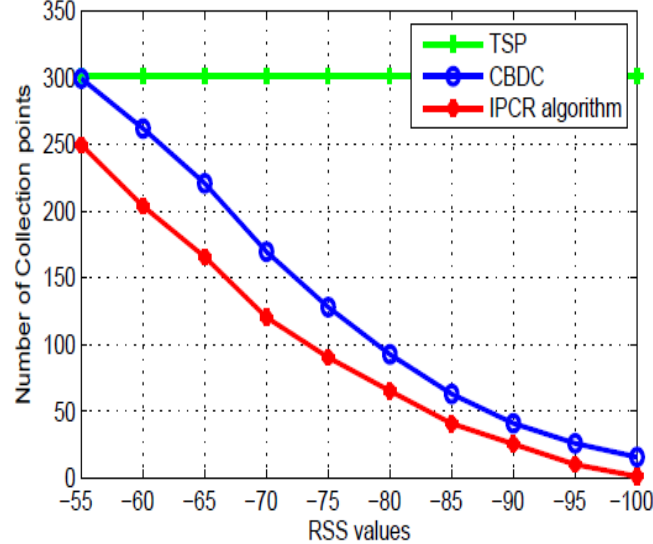

(b)

Fig. 4. The number of collection points of the IPCR, CBDC and TSP when (a) The number of sensor nodes is increased, and (b) the communication dth on range of sensor nodes is increased. 


\subsection{The number of collection points}

Collection points of the IPCR algorithm serve as stop stations where the mobile sink has to wait and collect data from neighboring nodes. Reducing the number of collection points definitely lead to improve the performance of data gathering through reducing network latency and increasing throughput as will be figured out in the subsequent sections. For this reason, the number of collection points was examined for the IPCR algorithm at increased number of sensor nodes (from 100 to 500 nodes) and at multiple levels of communication ranges, as shown in Figure 4a and b, respectively. The collection points for the Connectivity Based Data Collection (CBDC) algorithm in addition to the traditional TSP algorithm were also computed

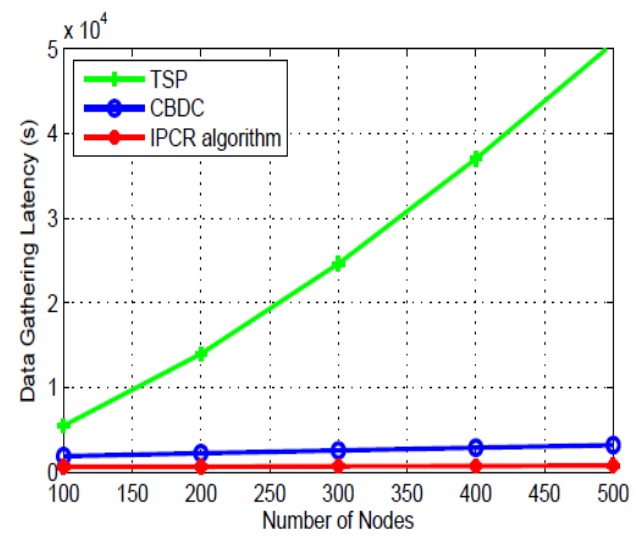

(a)

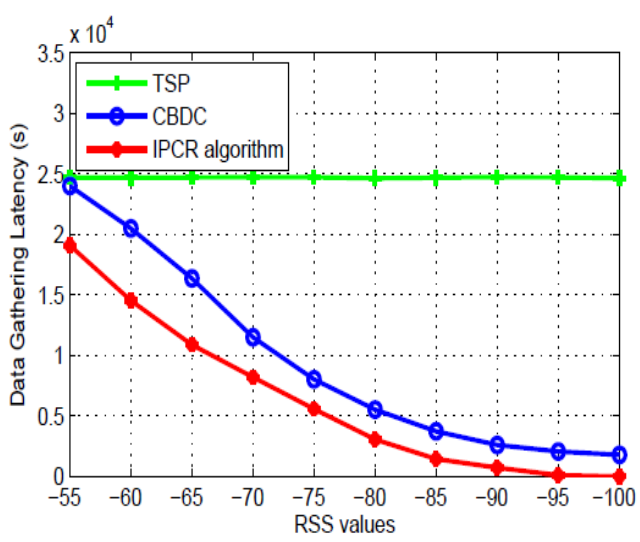

(b)

Figure 5. The data gathering latency the IPCR, CBDC and TSP when (a) The number of sensor nodes is increased, and (b) the communication dth on range of sensor nodes is increased.

Generally speaking, increasing the number of sensor nodes leads to increase the number of CPs as shown in Figure 4a. In contrast to the TSP algorithm where each node is considered as a collection point by itself, the CBDC and IPCR algorithms show insignificant increase of their collection points. More specifically, the IPCR outperforms the CBDC algorithm at the entire range of sensor nodes. In the same way, increasing the communication range of sensor nodes yield to reduce the number of collection points for the CBDC and IPCR algorithms, as shown in Figure $4 \mathrm{~b}$. While for the TSP algorithm the number of collection points is fixed at 300 which is the number of sensor nodes used in this simulation scenario. From this figure, it can be also observed that the lowest number of collection points is found with the IPCR algorithm as a function of increasing number of sensor nodes and increased communication ranges.

\subsection{Data Gathering Latency}

The data gathering latency is defined as the time needed until the mobile sink completes one collection round. Figure 5a and $\mathrm{b}$ represent the relationship between the data gathering latency versus the number of sensor nodes and the RSS values, respectively. As shown in Figure 5a, the Latency for IPCR algorithm is almost constant irrespective to the number of sensor nodes. Although the gap between the IPCR and CBDC algorithms is small, it expands as the number of sensor of nodes increases. On contrast, TSP algorithm demonstrates the highest latency. On the other hand, the data gathering latency is decreased as the communication range increased. This is quit apparent for the CBDC and IPCR algorithms. 
Notice that for the IPCR algorithm in addition to achieving the lowest latency, it almost reaches zero latency at communication ranges above $-90 \mathrm{dBm}$, (in other word, when the network connectivity is greater than 30\%). However, the data gathering latency due to the CBDC stabilizes at $1.8 \times 103$ seconds. Moreover, when the network connectivity is weak, the latency difference between the IPCR and CBDC is the maximum, while this difference declines as the connectivity is getting strengthen. Again, the latency of the TSP algorithm is constant since it is independent of the communication range.

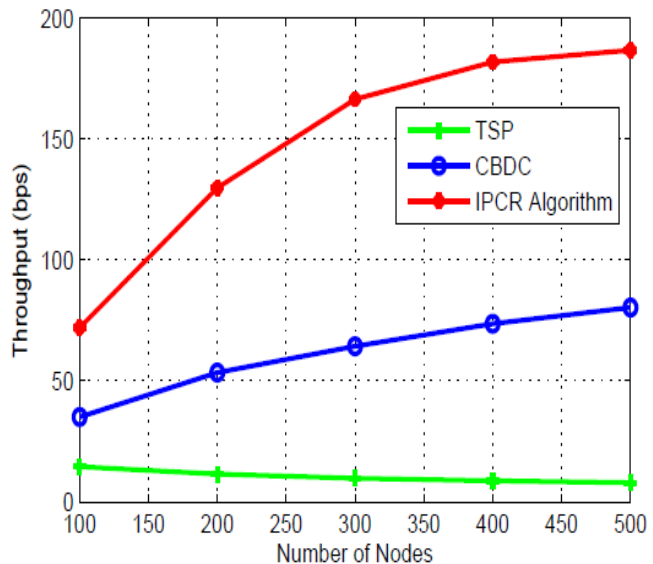

(a)

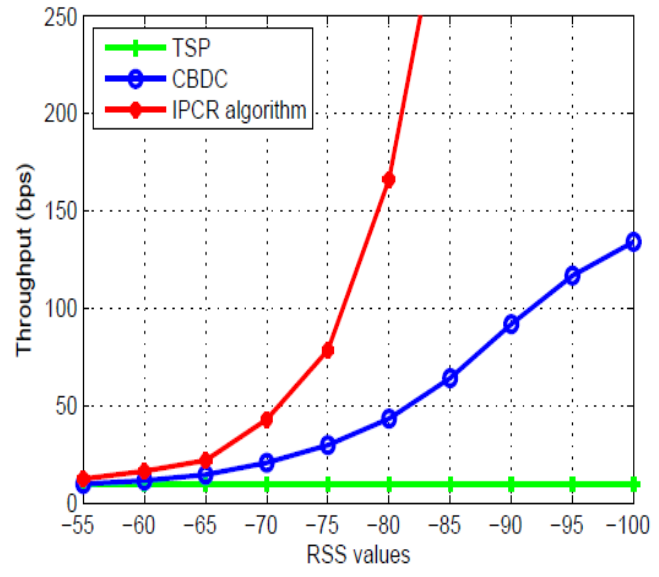

(b)

Figure 6. The network throughput of the IPCR, CBDC and TSP when (a) The number of sensor nodes is increased, and (b) the communication dth on range of sensor nodes is increased.

\subsection{Network Throughput}

To investigate the performance of these algorithms in term of Network throughput, the throughput of the three algorithms were evaluated and the results were displayed in Figure 6. In Figure 6a, the throughput as a function of number of sensor nodes is plotted. It is clear from this figure that the IPCR algorithms performs better throughput than the other algorithms. This is due to the fact that the IPCR algorithm has used lower number of collection points and therefore achieved the minimum latency. This is matched with equation (9), in which reducing the data collection latency results with increasing the network throughput. Although the CBDC shows lower throughput than the IPCR, it is throughput is increased as a function of increasing the number of sensor nodes. It is worth noting that at high number of sensor nodes the network throughputs are almost saturated despite the increase of nodes. This is due to the higher data collection latency found at large network sizes, as already explained in the previous section. Unsurprisingly, the throughput of the TSP algorithm slightly deteriorates as the number of sensor nodes increased. This is readily justified, since the data gathering latency in this algorithm is significantly high.

Furthermore, increasing the communication range of sensor nodes at a fixed number of sensor nodes also leads to increase the throughput of the sensor network, as shown in Figure 6b. The most interesting trend from this figure is that the IPCR algorithm presents an exponential increase of the network throughput as the connectivity of the sensor network becomes greater than $10 \%$. 
International Journal of Wireless \& Mobile Networks (IJWMN) Vol. 7, No. 6, December 2015

\section{Conclusion And Future Work}

This paper investigates an efficient mobile-based data gathering technique, referred to as Intersection Points of Communication Ranges (IPCR) algorithm. The key idea of the IPCR algorithm is to obtain an optimal trajectory of the mobile sink with the minimum number of collection points and single-hop communication. The simulation results demonstrated that the IPCR algorithm has exhibited a comparable result, which is almost identical, to the optimal Travel Sales-Man Problem (TSP) provided by the Mixed Integer Linear Programming (MILP). Moreover, the IPCR algorithm showed a superior performance in comparison with the Connectivity-Based Data Collection (CBDC) algorithm and the Travel Sales-Man Problem (TSP) algorithm and in terms of data gathering latency and network throughput.

As a part of future work, using multiple mobile elements could be investigated in an attempt to address some related problems such as buffering overflow and data gathering latency.

\section{REFERENCES}

[1] I. Akyildiz, W. Su, Y. Sankarasubramaniam and E. Cayirci, "Wireless sensor networks: a survey," IEEE Trans. Communication magazine, vol. 40, pp. 102-114, Aug. 2002

[2] Dan Li, Kerry D. Wong, Yu Hen Hu and Akbar M. Sayeed, "Detection, Classification and Tracking of Targets, ”IEEE Trans. Signal Processing Magazine, vol. 19, pp. 17-29, Mar.2002.

[3] Di Francesco M, Das S.K., "Data Collection in Wireless Sensor Networks with Mobile Elements: A survey," ACM Trans. on Sensor Networks (TOSN), Vol. 8(1), 2011.

[4] Al-Hasanat, A. I.; Matrouk, K. M.; Alasha'ary, H. A.;Alqadi, Z. A. "Connectivity-Based Clustering Algorithm for Data Gathering with Path-Constrained Mobile Sink inWireless Sensor Networks," Wireless Sensor Network Journal (WSN), Vol. 6(6): p.p 118-128, June 2014.

[5] L. Shangguan, L. Mai, J. Du, W. He, and H. Liu, "Energy efficient Heterogeneous Data Collection in Mobile Wireless Sensor Net-works," Proceedings of In 5th PMECT International Workshop colocated with the 20th International Conference on Computer Communication Networks (ICCCN'2011), 2011.

[6] B. Tang, J. Wang, X. Geng, Y. Zheng and J. Kim, "A Novel Reliable and Efficient Data Harvesting Mechanism in Wireless Sensor Networks with Path-Constrained Mobile Sink," Proceedings of SPDA / NT / ICE 2012 Conference,Hangzhou, China, Aug 2012.

[7] G. Xing, M. Li, T. Wang, W. Jia and J. Huang, "Efficient Rendezvous Algorithms for MobilityEnabled Wireless Sensor Networks," Proceeding of IEEE Transactions on Mobile Computing, vol .11, Jan. 2012.

[8] A. Somasundara, A. Ramamoorthy, and B. Srivastava, "Mobile Element Scheduling for Efficient Data Collection in Wireless Sensor Networks with Dynamic Deadlines," Proceedings of The 25th IEEE International Real-Time Systems Symposium (RTSS), pp. 296305, 2004.

[9] S. Gao, H. Zhang and Sajal K. Das, "Efficient Data Collection in Wireless Sensor Networks with Path-Constrained Mobile Sinks,” IEEE Trans. Mobile Computing, vol. 10, pp. 592-608, 2011.

[10] R. Shah, S. Roy, S. Jain, and W. Brunette, "Data MULEs: Modeling a Three-Tier Architecture for Sparse Sensor Networks," in The First IEEE Intl Workshop Sensor Network Protocols and Applications, pp. 30-41, 2003.

[11] Kansal, A., Somasundara, A., Jea, D., Srivastava, M., and Estrin, D., "Intelligent fluid infrastructure for embedded networks." in proceedings of the 2nd ACM International Conference on Mobile Systems, Applications, and Services (MobiSys2004), pp. 111-124, 2004.

[12] A. Somasundara, A. Kansal, D. Jea, D. Estrin, and M. Srivastava, "Controllably Mobile Infrastructure for Low Energy Embedded Networks," IEEE Trans. Mobile Computing, vol. 5, no. 8, pp. 958- 973, Aug. 2006.

[13] X. Li, "Collaborative localization with received-signal strength in wire-less sensor networks," IEEE Trans. Veh. Technol., vol. 56, pp. 3807-3817, 2007.

[14] Ming Ma, Yuanyuan Yang, "Data gathering in wireless sensor networks with mobile collectors," Parallel and Distributed Processing, 2008. IPDPS 2008. IEEE International Symposium on, 2008, p.p. $1-9$. 
International Journal of Wireless \& Mobile Networks (IJWMN) Vol. 7, No. 6, December 2015

[15] W.R., Heinzelman, A., Chandrakasan, \& H., Balakrishnan "Energy-Efficient Communication Protocol for Wireless Microsensor Networks", in Proceeding of the 33rd Hawaii International Conference on System Science, pages: 1-10. 2000

[16] S., Lindsey, \& S.C. Raghavendra "Pegasis: power-efficient gathering in sensor information systems" Proceedings of the IEEE Aerospace Conf. Montana: IEEE Computer Society, pp.1-6. 2002

[17] F., Flammini, A., Gaglione, F., Ottello, A., Pappalardo, C.Pragliola, \& A.Tedesco, "Towards Wireless Sensor Networks for Railway Infrastructure Monitoring,", Proceedings of Electrical Systems for Aircraft, Railway and Ship Propulsion (ESARS), pp.1-6.2010.

[18] P., Zhang, C., Sadler, S., Lyon, \& M.Martonosi, "Hardware design experiences in zebranet" ACM, Proceedings of the 2nd international conference on Embedded networked sensor systems (SenSys), pp. 227-238 Baltimore, MD, USA. 2004.

[19] S.R., Gandham, M., Dawande, R.Prakash, \& S. Venkatesan, "Energy Efficient Schemes for Wireless Sensor Networks with Multiple Mobile Base Stations”, IEEE, GLOBECOM, pp. 377-381. 2003.

[20] B., Alqaralleh, \& K. Almi' ani, "Data Gathering Scheme for Wireless Sensor Networks Using a Single Mobile Element", International Journal of Wireless \& Mobile Networks (IJWMN) Vol. 6, No. 3, pp 69-78, Jun 2014.

[21] N. Medhi, \& N. Sarma,. "Mobility Aided Cooperative MIMO Transmission in Wireless Sensor Networks", Elsevier ,Procedia Technology, vol. 6, pp 362 - 370. 2012

[22] R., Rajagopalan, \& P.K. Varshney, "Data aggregation techniques in sensor networks: A survey", Communications Surveys and Tutorials, IEEE, vol.8, pp. 48 - 63.2006. 